\title{
Hypergraph Clustering Based on PageRank
}

\author{
Yuuki Takai* \\ RIKEN AIP \\ Tokyo, Japan \\ yuuki.takai@riken.jp \\ Masahiro Ikeda \\ RIKEN AIP \\ Tokyo, Japan \\ masahiro.ikeda@riken.jp
}

\author{
Atsushi Miyauchi ${ }^{*}$ \\ University of Tokyo \\ Tokyo, Japan \\ miyauchi@mist.i.u-tokyo.ac.jp \\ Yuichi Yoshida \\ National Institute of Informatics \\ Tokyo, Japan \\ yyoshida@nii.ac.jp
}

\begin{abstract}
A hypergraph is a useful combinatorial object to model ternary or higher-order relations among entities. Clustering hypergraphs is a fundamental task in network analysis. In this study, we develop two clustering algorithms based on personalized PageRank on hypergraphs. The first one is local in the sense that its goal is to find a tightly connected vertex set with a bounded volume including a specified vertex. The second one is global in the sense that its goal is to find a tightly connected vertex set. For both algorithms, we discuss theoretical guarantees on the conductance of the output vertex set. Also, we experimentally demonstrate that our clustering algorithms outperform existing methods in terms of both the solution quality and running time. To the best of our knowledge, ours are the first practical algorithms for hypergraphs with theoretical guarantees on the conductance of the output set.
\end{abstract}

\section{CCS CONCEPTS}

- Information systems $\rightarrow$ Web mining; • Mathematics of computing $\rightarrow$ Spectra of graphs.

\section{KEYWORDS}

hypergraph, clustering, personalized PageRank, Laplacian

\section{INTRODUCTION}

Graph clustering is a fundamental task in network analysis, where the goal is to find a tightly connected vertex set in a graph. It has been used in many applications such as community detection [12] and image segmentation [11]. Because of its importance, graph clustering has been intensively studied, and a plethora of clustering methods have been proposed. See, e.g., [12] for a survey.

There is no single best criterion to measure the quality of a vertex set as a cluster; however, one of the most widely used is the notion of conductance. Roughly speaking, the conductance of a vertex set is small if many edges reside within the vertex set, whereas only a few edges leave it (see Section 3 for details). Many clustering methods have been shown to have theoretical guarantees on the conductance of the output vertex set [3-6, 10, 14, 18, 20, 30].

Although a graph is suitable to represent binary relations over entities, many relations, such as coauthorship, goods that are purchased together, and chemicals involved in metabolic reactions inherently comprise three or more entities. A hypergraph is a more natural object to model such relations. Indeed, if we represent coauthorship network by a graph, an edge only indicates the existence of co-authored paper, and information about co-authors in one paper is lost. However, hypergraphs do not lose that information.

As with graphs, hypergraph clustering is also an important task, and it is natural to use an extension of conductance for hypergraphs $[8,32]$ to measure the quality of a vertex set as a cluster. Although several methods have been shown to have theoretical guarantees on the conductance of the output vertex set $[8,16,32]$, none of them are efficient in practice.

To obtain efficient hypergraph clustering algorithms with a theoretical guarantee, we utilize the graph clustering algorithm developed by Andersen et al. [5]. Their algorithm is based on personalized PageRank (PPR), which is the stationary distribution of a random walk that jumps back to a specified vertex with a certain probability in each step of the walk. Roughly speaking, their algorithm first computes the PPR from a specified vertex, and then returns a set of vertices with high PPR values. They showed a theoretical guarantee on the conductance of the returned vertex set.

In this work, we propose two hypergraph clustering algorithms by extending Andersen et al.'s algorithm [5]. The first one is local in the sense that its goal is to find a vertex set of a small conductance and a bounded volume including a specified vertex. The second one is global in the sense that its goal is to find a vertex set of the smallest conductance.

Although there could be various definitions of random walk on hypergraphs and corresponding PPRs, we adopt the PPR recently introduced by Li and Milenkovic [22] using a hypergraph Laplacian $[8,32]$. Because the hypergraph Laplacian well captures the cut information of hypergraphs, the corresponding PPR can be used to find vertex sets with small conductance.

Because we can efficiently compute (a good approximation to) the PPR, our algorithms are efficient. Moreover, as with Andersen et al.'s algorithm [5], our algorithms have theoretical guarantees on the conductance of the output vertex set. To the best of our knowledge, no local clustering algorithm with a theoretical guarantee on the conductance of the output set is known for hypergraphs. Although, as mentioned previously, several algorithms have been proposed for global clustering for hypergraphs [8, 16, 32], we stress here that our global clustering algorithm is the first practical algorithm with a theoretical guarantee. 
We experimentally demonstrate that our clustering algorithms outperform other baseline methods in terms of both the solution quality and running time.

Our contribution can be summarized as follows.

- We propose local and global hypergraph clustering algorithms based on PPR for hypergraphs given by Li and Milenkovic [22].

- We give theoretical guarantees on the conductance of the vertex set output by our algorithms.

- Our algorithms are more efficient than existing algorithms with theoretical guarantees [8, 16, 32] (see Section 2 for the details).

- We experimentally demonstrate that our clustering algorithms outperform other baseline methods in terms of both the solution quality and running time.

This paper is organized as follows. We discuss related work in Section 2 and then introduce notions used throughout the paper in Section 3. After discussing basic properties of PPR for hypergraphs in Section 4, we introduce a key lemma (Lemma 4) that connects conductance and PPR in Section 5. On the basis of the key lemma, we show our local and global clustering algorithms in Sections 6 and 7, respectively. We discuss our experimental results in Section 8, and conclude in Section 9. In appendix, we give the proofs which could not be included in the main body because of the page restrictions.

\section{RELATED WORK}

As previously mentioned, even if we restrict our attention to graph clustering methods with theoretical guarantees on the conductance of the output vertex set, a plethora of methods have been proposed $[3-6,10,14,18,20,30]$. Some of them run in time nearly linear in the size of the output vertex set, i.e., we do not have to read the entire graph.

Although several hypergraph clustering algorithms have been proposed [2, 21, 23, 29, 34], most of them are merely heuristics and have no guarantees on the conductance of the output vertex set. Indeed, we are not aware of any local clustering algorithms with a theoretical guarantee, and to the best of our knowledge, there are only two global clustering algorithms with theoretical guarantees.

The first one $[8,32]$ is based on Cheeger's inequality for hypergraphs. Given a hypergraph $H=(V, E, w)$, it first computes an approximation $x \in \mathbb{R}^{V}$ to the second eigenvector of the normalized Laplacian $\mathcal{L}_{H}$ (see Section 3.3 for details), and then returns a sweep cut, i.e., a vertex set of the form $\{v \in V: \boldsymbol{x}(v)>\tau\}$ for some $\tau \in \mathbb{R}$. We can guarantee that the output vertex set has conductance roughly $O\left(\sqrt{\phi_{H} \log n}\right)$, where $\phi_{H}$ is the conductance of the hypergraph (see Section 3.2). However, to run the algorithm we need to approximately compute the second eigenvalue of the normalized Laplacian, which requires solving SDP; hence, is impractical.

The second algorithm was developed by Ikeda et al. [16]. Their algorithm is based on simulating the heat equation:

$$
\frac{d \rho_{t}}{d t} \in-\mathcal{L}_{H}\left(\boldsymbol{\rho}_{t}\right), \quad \boldsymbol{\rho}_{0}=\chi_{v}
$$

where $\chi_{v} \in \mathbb{R}^{V}$ is a vector with $\chi_{v}(u)=1$ if $u=v$ and 0 otherwise. Then, they showed that one of the sweep cuts of the form $\{v \in V$ : $\left.\rho_{t}(v)>\tau\right\}$ for some $\tau \in \mathbb{R}$ and $t \geq 0$, has conductance roughly $O\left(\sqrt{\phi_{H}}\right)$, under a mild condition. A drawback of their algorithm is that we need to calculate sweep cuts induced by $\rho_{t}$ for every $t \geq 0$, which cannot be efficiently implemented on Turing machines. Although our algorithm also computes sweep cuts, we need only consider one specific vector, i.e., the PPR.

If we do not need any theoretical guarantee on the quality of the output vertex set, we can think of a heuristic in which we first construct a graph from the input hypergraph $H=(V, E, w)$ and then apply a known clustering algorithm for graphs on the resulting graph, e.g., Andersen et al.'s algorithm [5]. There are two popular methods for constructing graphs:

Clique expansion [2, 34]: For each hyperedge $e \in E$ and each $u, v \in e$ with $u \neq v$, we add an edge $u v$ of weight $w(e)$ or $w(e) /\left(\begin{array}{c}|e| \\ 2\end{array}\right)$.

Star expansion [35]: For each hyperedge $e \in E$, we introduce a new vertex $v_{e}$, and then for each vertex $u \in e$, we add an edge $u v_{e}$ of weight $w(e)$ or $w(e) /|e|$.

The obvious drawback of the clique expansion is that it introduces $\Theta\left(k^{2}\right)$ edges for a hyperedge of size $k$, and hence the resulting graph is huge. The relation of these expansions and various Laplacians defined for hypergraphs [7, 28, 33] were discussed in [1].

Ghoshdastidar and Dukkipati [15] proposed a spectral method utilizing a tensor defined from the input hypergraph, and analyzed its performance when the hypergraph is generated from a planted partition model or a stochastic block model. A drawback of their method is that the hypergraph must be uniform, i.e., each hyperedge must be of the same size. Chien et al. [9] proposed another statistical method that also assumes uniformity of the hypergraph, and analyzed its performance on a stochastic block model.

Other iterative procedures for clustering hypergraphs have also been proposed [21, 23, 29]. However, these methods do not have any theoretical guarantee on the quality of the output vertex set.

\section{PRELIMINARIES}

We say that a vector $\boldsymbol{x} \in \mathbb{R}^{V}$ is a distribution if $\sum_{v \in V} \boldsymbol{x}(v)=1$ and $\boldsymbol{x}(v) \geq 0$ for every $v \in V$. For a vector $\boldsymbol{x} \in \mathbb{R}^{V}$ and a set $S \subseteq V$, we define $\boldsymbol{x}(S)=\sum_{v \in S} \boldsymbol{x}(v)$.

We often use the symbols $n$ and $m$ to denote the number of vertices and number of (hyper)edges of the (hyper)graph we are concerned with, which should be clear from the context.

\subsection{Personalized PageRank}

Let $G=(V, E, w)$ be a weighted graph, where $w: E \rightarrow \mathbb{R}_{+}$is a weight function over edges. For convenience, we define $w(u v)=0$ when $u v \notin E$. The degree of a vertex $u \in V$, denoted by $d_{u}$, is $\sum_{e \in E: u \in e} w(e)$. We assume $d_{u}>0$ for any $u \in V$. The degree matrix $D_{G} \in \mathbb{R}^{V \times V}$ and adjacency matrix $A_{G} \in \mathbb{R}^{V \times V}$ are defined as $D_{G}(u, u)=d_{u}$ and $D_{G}(u, v)=0$ for $u \neq v$, and $A_{G}(u, v)=w(u v)$. Lazy random walk is a stochastic process, which stays at the current vertex $u \in V$ with probability $1 / 2$ and moves to a neighbor $v \in V$ with probability $1 / 2 \cdot w(u v) / d_{u}$. The transition matrix of a lazy random walk can be written as $W_{G}=\left(I+A_{G} D_{G}^{-1}\right) / 2$.

For a graph $G=(V, E, w)$, a seed distribution $s \in \mathbb{R}^{V}$ and a parameter $\alpha \in(0,1]$, the personalized PageRank (PPR) is a vector $\operatorname{pr}_{\alpha}(s) \in \mathbb{R}^{V}$ [17] defined as the stationary distribution of a random walk that, with probability $\alpha$, jumps to a vertex $v \in V$ with probability $\boldsymbol{s}(v)$, and with the remaining probability $1-\alpha$, moves as the 
lazy random walk. We can rephrase $\operatorname{pr}_{\alpha}(s) \in \mathbb{R}^{V}$ as the solution to the following equation:

$$
\operatorname{pr}_{\alpha}(s)=\alpha s+(1-\alpha) W_{G} \operatorname{pr}_{\alpha}(s) .
$$

Using this equation, we can also define PPR for any vector $s \in \mathbb{R}^{V}$. For later convenience, we rephrase PPR in terms of Laplacian. For a graph $G=(V, E, w)$, its Laplacian $L_{G} \in \mathbb{R}^{V \times V}$ and (random-walk) normalized Laplacian $\mathcal{L}_{G} \in \mathbb{R}^{V \times V}$ are defined as $L_{G}=D_{G}-A_{G}$ and $\mathcal{L}_{G}=L_{G} D_{G}^{-1}=I-A_{G} D_{G}^{-1}$, respectively. Then, we have $W_{G}=I-\mathcal{L}_{G} / 2$. It is easy to check that the $\operatorname{PPR} \operatorname{pr}_{\alpha}(s)$ satisfies the equation

$$
\left(I+\frac{1-\alpha}{2 \alpha} \mathcal{L}_{G}\right) \operatorname{pr}_{\alpha}(s)=s
$$

\subsection{Hypergraphs}

In this section, we introduce several notions for hypergraphs. We note that these notions can also be used for graphs, because a hypergraph is a generalization of a graph.

Let $H=(V, E, w)$ be a (weighted) hypergraph, where $w: E \rightarrow$ $\mathbb{R}_{+}$is a weight function over hyperedges. The degree of a vertex $u \in V$, denoted by $d_{u}$, is $\sum_{e \in E: u \in e} w(e)$. We assume $d_{u}>0$ for any $u \in V$. The degree matrix $D_{H} \in \mathbb{R}^{V \times V}$ is defined as $D_{H}(u, u)=$ $d_{u}$ and $D_{H}(u, v)=0$ for $u \neq v$. Let $S \subseteq V$ be a vertex set and $\bar{S}=V \backslash S$. We define the set of the boundary hyperedges $\partial S$ by $\partial S=\{e \in E \mid e \cap S \neq \emptyset$ and $e \cap \bar{S} \neq \emptyset\}$. We define $S^{\circ}$ as the interior of $S$, that is, $v \in S^{\circ}$ if and only if $v \in S$ and $v \notin e$ for any $e \in \partial S$.

We define $\operatorname{cut}(S)$ as the total weight of hyperedges intersecting both $S$ and $\bar{S}$, that is, $\operatorname{cut}(S)=\sum_{e \in \partial S} w(e)$. We say that $H$ is connected if $\operatorname{cut}(S)>0$ for every $\emptyset \subsetneq S \subsetneq V$.

Next, we define the volume of a vertex set $S$ as $\operatorname{vol}(S)=\sum_{v \in V} d_{v}$. Then, we define the conductance of $S$ as

$$
\phi_{H}(S)=\frac{\operatorname{cut}(S)}{\min \{\operatorname{vol}(S), \operatorname{vol}(V \backslash S)\}} .
$$

We can say that a smaller $\phi_{H}(S)$ implies that the vertex set $S$ is a better cluster. The conductance of $H$ is $\phi_{H}=\min _{\emptyset \subsetneq S \subsetneq V} \phi_{H}(S)$.

Finally for $S \subseteq V$, we define the distribution $\pi_{S} \in \mathbb{R}^{V}$ as

$$
\pi_{S}(v)= \begin{cases}\frac{d_{v}}{\operatorname{vol}(S)} & \text { if } v \in S, \\ 0 & \text { otherwise. }\end{cases}
$$

We define $\chi_{v}$ as the indicator vector for $v \in V$, i.e., $\chi_{v}=\pi_{\{v\}}$.

\subsection{Personalized PageRank for hypergraphs}

In this section, we explain the notion of PPR for hypergraphs recently introduced by Li and Milenkovic [22].

We start by defining the Laplacian for hypergraphs and then use (1) to define PPR. For a hypergraph $H=(V, E, w)$, the (hypergraph) Laplacian $L_{H}: \mathbb{R}^{V} \rightarrow 2^{\mathbb{R}^{V}}$ of $H$ is defined as

$$
L_{H}(\boldsymbol{x})=\left\{\sum_{\boldsymbol{e} \in E} w(e) \boldsymbol{b}_{e} \boldsymbol{b}_{e}^{\top} \boldsymbol{x} \mid \boldsymbol{b}_{e} \in \underset{\boldsymbol{b} \in B_{e}}{\operatorname{argmax}} \boldsymbol{b}^{\top} \boldsymbol{x}\right\} \subseteq \mathbb{R}^{V},
$$

where $B_{e}$ for $e \in E$ is the convex hull of the vectors $\left\{\chi_{u}-\chi_{v} \mid\right.$ $u, v \in e\}[8,31,32]$. Note that $L_{H}$ is no longer a matrix, as opposed to the Laplacian of graphs. We then define the normalized Laplacian $\mathcal{L}_{H}: \mathbb{R}^{V} \rightarrow 2^{\mathbb{R}^{V}}$ as $\boldsymbol{x} \mapsto L_{H}\left(D_{H}^{-1} \boldsymbol{x}\right)$. We also define $A_{H}: \mathbb{R}^{V} \rightarrow$

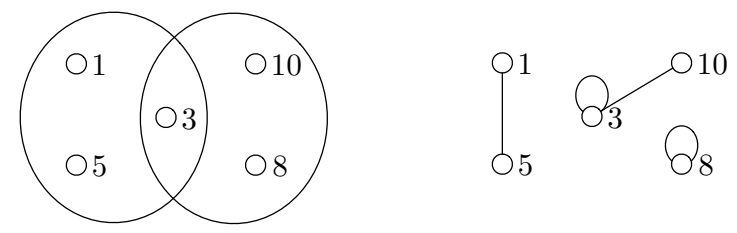

Figure 1: An example of a hypergraph $H$ and the graph $H_{x}$. The values next to vertices represent a vector $x \in \mathbb{R}^{V}$.

$2^{\mathbb{R}^{V}}$ as $A_{H}=D_{H}-L_{H}$, or more formally, $\boldsymbol{x} \mapsto\left\{D_{H} \boldsymbol{x}-\boldsymbol{y} \mid \boldsymbol{y} \in\right.$ $\left.L_{H}(\boldsymbol{x})\right\}$ and $W_{H}: \mathbb{R}^{V} \rightarrow 2^{\mathbb{R}^{V}}$ as $\boldsymbol{x} \mapsto\left(\boldsymbol{x}+A_{H}\left(D_{H}^{-1} \boldsymbol{x}\right)\right) / 2$. Note that when $H$ is a graph, each of $L_{H}(\boldsymbol{x}), \mathcal{L}_{H}(\boldsymbol{x}), A_{H}(\boldsymbol{x})$, and $W_{H}(\boldsymbol{x})$ defined above is a singleton consisting of the vector obtained by applying the corresponding matrix to $\boldsymbol{x}$.

To understand how the Laplacian $L_{H}$ acts on $x$ when $H$ is not a graph, suppose that the entries of the vector $x \in \mathbb{R}^{V}$ are pairwise distinct. Then for each hyperedge $e \in E$, we have $\boldsymbol{b}_{e}=\chi_{s_{e}}-\chi_{t_{e}}$, where $s_{e}=\operatorname{argmax}_{v \in e} \boldsymbol{x}(v)$ and $t_{e}=\operatorname{argmin}_{v \in e} \boldsymbol{x}(v)$. Then, we construct a graph $H_{\boldsymbol{x}}=\left(V, E_{\boldsymbol{x}}, w_{\boldsymbol{x}}\right)$ on the vertex set $V$ by, for each hyperedge $e \in E$, adding the edge $s_{e} t_{e}$ of weight $w(e)$ and the self-loop of weight $w(e)$ at $v$ for each $v \in e \backslash\left\{s_{e}, t_{e}\right\}$. Note that the degree of a vertex is unchanged between $H$ and $H_{x}$. Then, $L_{H}(\boldsymbol{x})=\left\{L_{H_{\boldsymbol{x}}} \boldsymbol{x}\right\}$, where $L_{H_{\boldsymbol{x}}}$ is the Laplacian of the graph $H_{\boldsymbol{x}}$. An example of the construction of $H_{x}$ is shown in Figure 1.

For general $x \in \mathbb{R}^{V}$, there could be many choices for $\boldsymbol{b}_{e}(e \in E)$ in (2). For any choice of $\boldsymbol{b}_{e} \in \operatorname{argmax}_{\boldsymbol{b} \in B_{e}} \boldsymbol{b}^{\top} \boldsymbol{x}$ for each $e \in E$, we can define a graph $H_{\boldsymbol{x}}=\left(V, E_{\boldsymbol{x}}, w_{\boldsymbol{x}}\right)$ so that $L_{H_{\boldsymbol{x}}}=\sum_{e \in E} w(e) \boldsymbol{b}_{\boldsymbol{e}} \boldsymbol{b}_{\boldsymbol{e}}^{\top}$. We remark that $w_{x}$ can output negative values naturally. However, it is easy to see that there exists a graph $\widetilde{H}_{\boldsymbol{x}}=\left(V, \widetilde{E}_{\boldsymbol{x}}, \widetilde{w}_{\boldsymbol{x}}\right)$ such that $\widetilde{w}_{\boldsymbol{x}}$ is non-negative and $\mathcal{L}_{\widetilde{H}_{\boldsymbol{x}}} \boldsymbol{x}=\mathcal{L}_{H_{\boldsymbol{x}}} \boldsymbol{x}$. This $\widetilde{H}_{\boldsymbol{x}}$ is the same as the graph introduced in [8]. In the following, we assume that when we choose a graph $H_{\boldsymbol{x}}$, the weight function $w_{\boldsymbol{x}}$ is non-negative.

Following (1), we define the PPR $\operatorname{pr}_{\alpha}(s)$ of $H$ as a solution (if exists) to

$$
\left(I+\frac{1-\alpha}{2 \alpha} \mathcal{L}_{H}\right)(\boldsymbol{x}) \ni \boldsymbol{s} .
$$

We can observe that $\operatorname{pr}_{\alpha}(s)=s$ when $\alpha=1$ and $\operatorname{pr}_{\alpha}(s) \rightarrow \pi_{V}$ when $\alpha \rightarrow 0$. In Section 4, we show that (3) indeed has a solution and it is unique for any $s \in \mathbb{R}^{V}$ and $\alpha \in(0,1]$.

\subsection{Sweep cuts}

For a hypergraph $H=(V, E, w)$ and a vector $\boldsymbol{x} \in \mathbb{R}^{V}$, we say that a set of the form $\left\{v \in V \mid \boldsymbol{x}(v) / d_{v}>\tau\right\}$ for some $\tau \in \mathbb{R}$ is a sweep cut induced by $\boldsymbol{x}$.

To discuss local clustering, we consider the minimum conductance of a sweep cut with a restriction on the volume. First, we order the vertices $v_{1}, v_{2}, \ldots, v_{n}$ in $V$ so that

$$
\frac{\boldsymbol{x}\left(v_{1}\right)}{d_{v_{1}}} \geq \frac{\boldsymbol{x}\left(v_{2}\right)}{d_{v_{2}}} \geq \cdots \geq \frac{\boldsymbol{x}\left(v_{n}\right)}{d_{v_{n}}} .
$$

Then, we can represent sweep cuts explicitly as

$$
S_{j}^{x}=\left\{v_{1}, v_{2}, \ldots, v_{j}\right\} \quad(j=1,2, \ldots, n) .
$$


We set $S_{0}^{\boldsymbol{x}}=\emptyset$ for convenience. For $\mu \in(0,1 / 2]$, let $\ell_{\mu}$ be the unique integer such that $\operatorname{vol}\left(S_{\ell_{\mu}-1}^{x}\right)<\mu \cdot \operatorname{vol}(V) \leq \operatorname{vol}\left(S_{\ell_{\mu}}^{x}\right)$. Then, we define $\phi_{H}^{\mu}(\boldsymbol{x})$ as $\phi_{H}^{\mu}(\boldsymbol{x})=\min \left\{\phi_{H}\left(S_{j}^{\boldsymbol{x}}\right) \mid j=1,2, \ldots, \ell_{\mu}\right\}$.

\section{PERSONALIZED PAGERANK FOR HYPERGRAPHS}

In this section, we discuss basic properties and the computational aspects of the PPR for hypergraphs. The proofs of the theorem and propositions in this section are given in Appendix A.

\subsection{Basic properties}

Here we introduce some properties of the PPR. We first argue that (3) has a solution and it is unique for any $s \in \mathbb{R}^{V}$ and $\alpha \in(0,1]$.

Theorem 1. For any $s \in \mathbb{R}^{V}$ and $\alpha \in(0,1]$, there exists a unique solution to (3), and hence the PPR $\mathbf{p r}_{\alpha}(\mathbf{s})$ is well-defined. Moreover, if $\boldsymbol{s}$ is a distribution, then so is $\mathbf{p r}_{\alpha}(\mathbf{s})$.

We next investigate the continuity of $\operatorname{pr}_{\alpha}(s)$ with respect to $\alpha$.

Proposition 2. For any $s \in \mathbb{R}^{V}$, the $P P R \operatorname{pr}_{\alpha}(s)$ is continuous with respect to $\alpha \in(0,1]$.

The following proposition shows that the value of PPR at each vertex can be bounded by the value of the distribution $\pi_{V}$.

Proposition 3. Let $H=(V, E, w)$ be a connected hypergraph. Then for any $\alpha \in(0,1]$ and $a$ vertex $u \in V$, we have

$$
\operatorname{pr}_{\alpha}\left(\chi_{u}\right)(v) \begin{cases}\geq \pi_{V}(u) & \text { if } v=u, \\ \leq \pi_{V}(v) & \text { if } v \neq u\end{cases}
$$

\subsection{Computation}

It is not difficult to show that we can compute the PPR in polynomial time by directly solving (3) using the techniques developed in [13] However, their algorithm involves the ellipsoid method, which is inefficient in practice. Hence, we consider a different approach here.

Lemma 15 in Appendix A indicates that $\operatorname{pr}_{\alpha}(s)$ is a stationary solution to the following differential equation:

$$
\frac{d \boldsymbol{\rho}_{t}}{d t} \in \beta\left(\boldsymbol{s}-\boldsymbol{\rho}_{t}\right)-(1-\beta) \mathcal{L}_{H}\left(\boldsymbol{\rho}_{t}\right),
$$

where $\beta:=2 \alpha /(1+\alpha)$. From the fact that $\mathcal{L}_{H}$ is a maximal monotone operator [16], it is easy to show that the operator

$$
\boldsymbol{x} \mapsto \beta(s-\boldsymbol{x})-(1-\beta) \mathcal{L}_{H}(\boldsymbol{x})
$$

is also maximally monotone. This implies that (4) has a unique solution for any given initial state $\boldsymbol{\rho}_{0}$. Moreover, if the hypergraph $H$ is connected, then the solution $\boldsymbol{\rho}_{t}$ of (4) converges to the stationary solution $\operatorname{pr}_{\alpha}(s)$ as $t$ tends to infinity. Hence, we can compute the PPR by simulating (4).

It is not difficult to show that we can simulate (4) on a Turing machine with a certain error guarantee by time discretization using the techniques in [16]. In our experiments (Section 8), however, we simply simulated it using the Euler method for practical efficiency.

We remark that simulating (4) by the Euler method is equivalent to running the gradient descent algorithm on the convex function

$$
Q(\widetilde{\boldsymbol{x}})=\frac{\beta}{2}\|\widetilde{\boldsymbol{x}}-\widetilde{\boldsymbol{s}}\|^{2}+(1-\beta) \widetilde{\boldsymbol{x}}^{\top} \overline{\mathcal{L}}_{H}(\widetilde{\boldsymbol{x}}),
$$

where $\widetilde{\boldsymbol{s}}=D^{-1 / 2} \boldsymbol{s}$ and $\overline{\mathcal{L}}_{H}=D^{-1 / 2} L_{H} D^{-1 / 2}$. To see this, note that the operator (5) can be rewritten as the operator $\boldsymbol{x} \mapsto-D^{1 / 2} \partial Q(\widetilde{\boldsymbol{x}})$, where $\widetilde{\boldsymbol{x}}=D^{-1 / 2} \boldsymbol{x}$ and $\partial Q(\widetilde{\boldsymbol{x}})$ is the sub-differential of $Q(\widetilde{\boldsymbol{x}})$ with respect to the variable $\tilde{x}$. Then, the differential inclusion (4) can be written as

$$
\frac{d \widetilde{\boldsymbol{\rho}}_{t}}{d t} \in-\partial Q\left(\widetilde{\boldsymbol{\rho}}_{t}\right)
$$

for $\widetilde{\rho}_{t}=D^{-1 / 2} \boldsymbol{\rho}_{t}$ and by [27, Theorem 6.9], the Euler sequence $\left\{\tilde{\boldsymbol{\rho}}_{t_{i}}\right\}_{i=1}^{\infty}$ for (6) converges to an optimal solution $\widetilde{\boldsymbol{\rho}}_{\infty}$, i.e., a solution satisfying $0 \in \partial Q\left(\widetilde{\boldsymbol{\rho}}_{\infty}\right)$, under the assumption $\sum\left\|\widetilde{\boldsymbol{\rho}}_{t_{i+1}}-\widetilde{\boldsymbol{\rho}}_{t_{i}}\right\|^{2}<\infty$. Then, $\boldsymbol{\rho}_{\infty}=D^{1 / 2} \widetilde{\boldsymbol{\rho}}_{\infty}$ is nothing but the PPR $\operatorname{pr}_{\alpha}(\boldsymbol{s})$.

\section{PERSONALIZED PAGERANK AND CONDUCTANCE}

In this section, we introduce the following lemma, which extends the result in [5] to hypergraphs and to the local setting. We prove this lemma in Appendix B.

Lemma 4. Let $s \in \mathbb{R}^{V}$ be a distribution and $\mu \in(0,1 / 2]$. If there is a vertex set $S \subseteq V$ and a constant $\delta \geq 4 / \sqrt{\operatorname{vol}(V)}$ such that $\operatorname{vol}(S) / \operatorname{vol}(V) \leq \mu$ and $\operatorname{pr}_{\alpha}(s)(S)-\pi_{V}(S)>\delta$, then we have

$$
\phi_{H}^{\mu}\left(\mathbf{p r}_{\alpha}(s)\right)<\sqrt{\frac{24 \alpha \log (4 / \delta)}{\delta}} .
$$

This lemma indicates that if the $\mathrm{PPR} \mathrm{pr}_{\alpha}(s)$ is far from the stationary distribution $\pi_{V}$ on some vertex set $S$ with a small volume, then there is a sweep cut induced by $\operatorname{pr}_{\alpha}(s)$ with small volume and conductance. Thus, we can reduce the clustering problem to showing the existence of a vertex set of a large PPR mass.

\section{LOCAL CLUSTERING}

For a hypergraph $H=(V, E, w), v \in V$, and $\mu \in(0,1 / 2]$, we define

$$
\phi_{H, v}^{\mu}=\min _{\substack{0 \subsetneq C \subsetneq V, v \in C, \operatorname{vol}(C) \leq \mu \cdot \operatorname{vol}(V)}} \phi_{H}(C) .
$$

In the local clustering problem, given a hypergraph $H=(V, E, w)$ and a vertex $v \in V$, the goal is to find a vertex set containing $v$ whose volume is bounded by $\mu \cdot \operatorname{vol}(V)$ with a conductance close to $\phi_{H, v}^{\mu}$. Let $C_{v}^{*}$ be an arbitrary minimizer of (8). We define $w_{\min }=$ $\min _{e \in E}\{w(e)\}$, and $w_{\max }=\max _{e \in E}\{w(e)\}$. In this section, we show the following:

Theorem 5. Let $H=(V, E, w)$ be a hypergraph, $v \in V$ be a vertex with $v \in\left(C_{v}^{*}\right)^{\circ}$, and $\mu \in(0,1 / 2]$. Our algorithm (Algorithm 1) returns a vertex set $S \subseteq V$ with $v \in S, \operatorname{vol}(S) \leq \mu \cdot \operatorname{vol}(V)+\max _{v \in V}\left\{d_{v}\right\}$, and $\phi_{H}(S)=O\left(\sqrt{\phi_{H, v}^{\mu}}\right)$ in $O\left(\left(T_{H}+\sum_{e \in E}|e|\right) \log \left(w_{\max } \sum_{e \in E}|e| / w_{\min }\right)\right)$ time, where $T_{H}$ is the time to compute PPR on $H$.

We stress here again that Theorem 5 is the first local clustering algorithm with a theoretical guarantee on the conductance. If we use the Euler method to compute PPR, then we have $T_{H}=$ $O\left(\sum_{e \in E}|e| / \Delta\right)$, where $\Delta$ is the time span for one step. Hence, the total time complexity is $O\left(\sum_{e \in E}|e| \log \left(w_{\max } \sum_{e \in E}|e| / w_{\min }\right) / \Delta\right)$. We also remark that we can relax the assumption $v \in\left(C_{v}^{*}\right)^{\circ}$, if we admit using the values of the PPR in the assumption. 


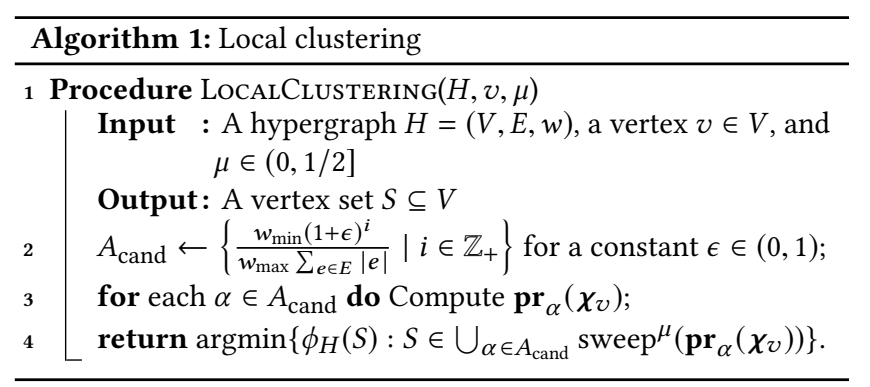

\subsection{Algorithm}

We describe our algorithm for local clustering. Given a hypergraph $H=(V, E, w)$ and a vertex $v \in V$, we first construct a set $A_{\text {cand }}=$ $\left\{\frac{w_{\min }(1+\epsilon)^{i}}{w_{\max } \sum_{e \in E}|e|} \mid i \in \mathbb{Z}_{+}\right\} \cap[0,1]$ for a constant $\epsilon \in(0,1)$. As the minimum conductance of a set in a connected hypergraph is lowerbounded by $\frac{w_{\min }}{w_{\max } \sum_{e \in E}|e|}$, we can guarantee that there exists $\alpha \in$ $A_{\text {cand }}$ such that $\alpha \leq \phi_{H, v}^{\mu} \leq(1+\epsilon) \alpha$. Then for each $\alpha \in A_{\text {cand }}$, we compute the PPR $\mathbf{p r}_{\alpha}\left(\chi_{v}\right)$. Finally, we return the set $S$ with the minimum conductance in

$$
\bigcup_{\alpha \in A_{\text {cand }}} \operatorname{sweep}^{\mu}\left(\mathbf{p r}_{\alpha}\left(\chi_{v}\right)\right)
$$

where sweep ${ }^{\mu}(\boldsymbol{x})=\left\{S_{1}^{\boldsymbol{x}}, \ldots, S_{\ell_{\mu}}^{\boldsymbol{x}}\right\}$. Proposition 3 implies that the normalized PPR value $\operatorname{pr}_{\alpha}\left(\chi_{v}\right)(v) / d_{v}$ takes the maximum value at $v$; hence the returned set $S$ includes the specified vertex $v$. The pseudocode of our local clustering algorithm is given in Algorithm 1.

\subsection{Leak of personalized PageRank}

As we want to apply Lemma 4 on $C_{v}^{*}$, we need to bound the amount of the leak of PPR from $C_{v}^{*}$. To this end, we start by showing the following upper bound.

Lemma 6. Let $C \subseteq V$ be a vertex set, $w \in C$, and $H_{p}=\left(V, E_{p}, w_{p}\right)$ be the graph defined for $\boldsymbol{p}=\operatorname{pr}_{\alpha}\left(\chi_{w}\right)$ as shown in Section 3.3. Then for any $\alpha \in(0,1]$, we have

$$
\boldsymbol{p}(\bar{C}) \leq \frac{1-\alpha}{2 \alpha} \sum_{u \in C} \sum_{v \in \bar{C}} \frac{w_{p}(u v)}{d_{u}} \boldsymbol{p}(u),
$$

Proof. By the definition of $H_{p}$, the PPR $p$ satisfies

$$
\begin{aligned}
\boldsymbol{p}(\bar{C}) & =\alpha \boldsymbol{\chi}_{w}(\bar{C})+(1-\alpha) W_{H_{p}}(\boldsymbol{p})(\bar{C}) \\
& =(1-\alpha) \boldsymbol{p}(\bar{C})+(1-\alpha) \frac{1}{2}\left(A_{H_{p}} D_{H_{p}}^{-1}-I\right) \boldsymbol{p}(\bar{C}) .
\end{aligned}
$$

Here, the second equality follows from $\chi_{w}(\bar{C})=0$ and $W_{H_{p}}=$ $I+\left(A_{H_{p}} D_{H_{p}}^{-1}-I\right) / 2$. Then, we have

$$
\boldsymbol{p}(\bar{C})=\frac{1-\alpha}{2 \alpha}\left(A_{H_{p}} D_{H_{p}}^{-1}-I\right) \boldsymbol{p}(\bar{C}) .
$$

For a subset $S \subseteq V$, we define two sets of ordered pairs of vertices: $\operatorname{in}(S)=\{(u, v) \in V \times S\}$ and $\operatorname{out}(S)=\{(u, v) \in S \times V\}$. For an ordered pair $(u, v) \in V \times V$, we set $\boldsymbol{p}(u, v):=\frac{w_{\boldsymbol{p}}(u v)}{d_{u}} \boldsymbol{p}(u)$. Then, the term $\left(A_{H_{\boldsymbol{p}}} D_{H_{\boldsymbol{p}}}^{-1}-I\right)(\boldsymbol{p})(\bar{C})$ can be rewritten as

$$
\begin{aligned}
& \left(A_{H_{p}} D_{H_{p}}^{-1}-I\right)(\boldsymbol{p})(\bar{C})=\sum_{u \in \bar{C}} \sum_{v \in V} \frac{w_{\boldsymbol{p}}(u v)}{d_{v}} \boldsymbol{p}(v)-\sum_{u \in \bar{C}} \boldsymbol{p}(u) \\
& =\sum_{u \in \bar{C}} \sum_{v \in V} \frac{w_{\boldsymbol{p}}(u v)}{d_{v}} \boldsymbol{p}(v)-\sum_{u \in \bar{C}} \sum_{v \in V} \frac{w_{\boldsymbol{p}}(u v)}{d_{u}} \boldsymbol{p}(u) \\
& =\sum_{(u, v) \in \operatorname{in}(\bar{C}) \mid \operatorname{out}(\bar{C})} \boldsymbol{p}(u, v)-\sum_{(u, v) \in \operatorname{out}(\bar{C}) \operatorname{in}(\bar{C})} \boldsymbol{p}(u, v) \\
& \leq \sum_{(u, v) \in \operatorname{in}(\bar{C}) \text { out }(\bar{C})} \boldsymbol{p}(u, v)=\sum_{u \in C} \sum_{v \in \bar{C}} \frac{w_{p}(u v)}{d_{u}} \boldsymbol{p}(u) .
\end{aligned}
$$

The claim follows by combining (9) and (10).

Next, we show that the amount of the leak of PPR from a vertex set $C$ can be bounded using its conductance.

Lemma 7. Let $C \subseteq V$ be a vertex set with $\operatorname{vol}(C) \leq \operatorname{vol}(V) / 2$. Then, for any $\alpha \in(0,1]$ and a vertex $v \in C^{\circ}$, we have

$$
\operatorname{pr}_{\alpha}\left(\chi_{v}\right)(\bar{C}) \leq \frac{1}{4 \alpha} \phi_{H}(C)
$$

Proof. Let $\boldsymbol{p}=\operatorname{pr}_{\alpha}\left(\chi_{v}\right)$. By Lemma 6, we have

$$
\frac{2 \alpha}{1-\alpha} p(\bar{C}) \leq \sum_{u^{\prime} \in C} \sum_{v^{\prime} \in \bar{C}} \frac{w_{p}\left(u^{\prime} v^{\prime}\right)}{d_{u^{\prime}}} p\left(u^{\prime}\right)
$$

As $v \in C$, by Proposition 3, the RHS of (11) is at most

$$
\sum_{u^{\prime} \in C} \sum_{v^{\prime} \in \bar{C}} \frac{w_{\boldsymbol{p}}\left(u^{\prime} v^{\prime}\right)}{d_{u^{\prime}}} \frac{d_{u^{\prime}}}{\operatorname{vol}(V)}+\sum_{v^{\prime} \in \bar{C}} \frac{w_{\boldsymbol{p}}\left(v v^{\prime}\right)}{d_{v}} \boldsymbol{p}(v)-\sum_{v^{\prime} \in \bar{C}} \frac{w_{\boldsymbol{p}}\left(v v^{\prime}\right)}{d_{v}} \frac{d_{v}}{\operatorname{vol}(V)} \text {. }
$$

From the assumption $v \in C^{\circ}, w_{p}\left(v v^{\prime}\right)$ is zero for any $v^{\prime} \in \bar{C}$. Hence, we have

$$
\begin{aligned}
\boldsymbol{p}(\bar{C}) & \leq \frac{1-\alpha}{2 \alpha} \sum_{u^{\prime} \in C} \sum_{v^{\prime} \in \bar{C}} \frac{w_{p}\left(u^{\prime} v^{\prime}\right)}{\operatorname{vol}(V)} \leq \frac{1-\alpha}{2 \alpha} \frac{\operatorname{vol}(C)}{\operatorname{vol}(V)} \phi_{H}(C) \\
& \leq \frac{1-\alpha}{4 \alpha} \phi_{H}(C) \leq \frac{1}{4 \alpha} \phi_{H}(C)
\end{aligned}
$$

The second inequality follows from

$$
\sum_{u^{\prime} \in C} \sum_{v^{\prime} \in \bar{C}} w_{p}\left(u^{\prime} v^{\prime}\right) \leq \sum_{e \in \partial C} w(e)
$$

\subsection{Proof of Theorem 5}

First, we discuss the conductance of the output vertex set.

LemmA 8. Let $\mu \in(0,1 / 2]$. Suppose $v \in\left(C_{v}^{*}\right)^{\circ}$. Then for any $\epsilon \in(0,1)$ and $\alpha \in(0,1]$ with $\alpha \leq \phi_{H}\left(C_{v}^{*}\right) \leq(1+\epsilon) \alpha$, we have

$$
\phi_{H}^{\mu}\left(\mathbf{p r}_{\alpha}\left(\chi_{v}\right)\right)<\frac{8}{\sqrt{3-\epsilon-4 \mu}} \sqrt{6 \phi_{H}\left(C_{v}^{*}\right) \log \left(\frac{2}{3-\epsilon-4 \mu}\right)} .
$$

Proof. Let $C=C_{v}^{*}$ and $\boldsymbol{p}=\operatorname{pr}_{\alpha}\left(\chi_{v}\right)$. To apply Lemma 4, we want to lower-bound $\boldsymbol{p}(C)-\boldsymbol{\pi}_{V}(C)$. By the fact that the PPR $\boldsymbol{p}$ is a distribution by Theorem 1 and Lemma 7 for $\alpha \geq \phi_{H}(C) /(1+\epsilon)$, we have $\boldsymbol{p}(C)-\boldsymbol{\pi}_{V}(C)=1-\boldsymbol{p}(\bar{C})-\boldsymbol{\pi}_{V}(C) \geq 1-(1+\epsilon) / 4-\mu=$ 


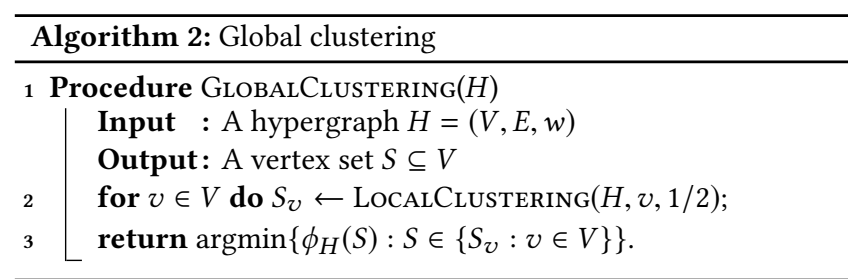

$(3-\epsilon-4 \mu) / 4$. Applying Lemma 4 with $C=C_{v}^{*}, \delta=(3-\epsilon-4 \mu) / 4$, and $\alpha \leq \phi_{H}(C)$, we obtain

$$
\phi_{H}^{\mu}(\boldsymbol{p})<\frac{8}{\sqrt{3-\epsilon-4 \mu}} \sqrt{6 \phi_{H, v}^{\mu} \log \left(\frac{2}{3-\epsilon-4 \mu}\right)} .
$$

Proof of Theorem 5. The guarantee on conductance holds by Lemma 8 as some $\alpha \in A_{\text {cand }}$ satisfies the condition of Lemma 8 with $\epsilon \in(0,1)$.

Now, we analyze the time complexity. As the size of $\left|A_{\text {cand }}\right|=$ $O\left(\log \left(w_{\max } \sum_{\boldsymbol{e} \in E}|e| / w_{\min }\right)\right)$, it takes $O\left(T_{H} \log \left(w_{\max } \sum_{\boldsymbol{e} \in E}|e| / w_{\min }\right)\right)$ time to compute all the PPRs. Computing the conductance values of the sweep cuts induced by a particular vector takes $O\left(\sum_{e \in E}|e|\right)$ time. Hence, Line 4 takes $O\left(\sum_{e \in E}|e| \log \left(w_{\max } \sum_{e \in E}|e| / w_{\min }\right)\right)$ time. Combining those, we obtain the claimed time complexity.

\section{GLOBAL CLUSTERING}

In the global clustering problem, given a hypergraph $H$, the goal is to find a vertex set with conductance close to $\phi_{H}$. In this section, we show the following:

Theorem 9. Let $H=(V, E, w)$ be a hypergraph. Our algorithm (Algorithm 2) returns a vertex set $S \subseteq V$ with $\phi_{H}(S)=O\left(\sqrt{\phi_{H}}\right)$ in $O\left(\left(T_{H}+\sum_{e \in E}|e|\right) n \log \left(w_{\max } \sum_{e \in E}|e| / w_{\min }\right)\right)$ time, where $T_{H}$ is the time to compute PPR on $H$.

\subsection{Algorithm}

Our global clustering algorithm is quite simple. That is, given a hypergraph $H=(V, E, w)$, we simply call LocalCLustering $(H, v, 1 / 2)$ for every $v \in V$, and then return the best returned set in terms of conductance. The pseudocode is given in Algorithm 2.

\subsection{Leak of personalized PageRank}

Let $C^{*}$ be an arbitrary set of conductance $\phi_{H}$. As we want to apply Lemma 4 for $\mu=1 / 2$ on $\phi_{H}$, we need to bound the amount of the leak of PRR from $\phi_{H}$. The following lemma is a counterpart to Lemma 7 for the global setting.

Lemma 10. Suppose that $\alpha \in(0,1]$ and $C \subseteq V$ satisfy

$$
\left(\sum_{w \in C} \pi_{C}(w) \mathbf{p r}_{\alpha}\left(\chi_{w}\right)\right)(v) \leq \pi_{C}(v)
$$

for every $v \in C \backslash C^{\circ}$. Then we have

$$
\sum_{w \in C} \pi_{C}(w) \operatorname{pr}_{\alpha}\left(\chi_{w}\right)(\bar{C}) \leq \frac{\phi_{H}(C)}{2 \alpha} .
$$

The assumption (13) means that the expected PPR of a boundary vertex $v \in C \backslash C^{\circ}$ is at most its probability mass in the distribution $\boldsymbol{\pi}_{C}$. We note that (13) always holds as an equality when $H$ is a graph. We discuss two sufficient conditions of this assumption in Lemmas 13 and 14 in Section 7.4. These conditions show that the assumption of Lemma 10 is quite mild.

Proof of Lemma 10. We set $\boldsymbol{p}_{w}=\operatorname{pr}_{\alpha}\left(\chi_{w}\right)$ and let $w_{0} \in C$ be an arbitrary vertex that maximizes $\sum_{v \in \bar{C}} w_{p_{w}}(u v)$. Then, by Lemma 6, we have

$$
\left(A_{H} D_{H}^{-1}-I\right)\left(\boldsymbol{p}_{w}\right)(\bar{C}) \leq \sum_{u \in C} \sum_{v \in \bar{C}} \frac{w_{p_{w_{0}}}(u v)}{d_{u}} \boldsymbol{p}_{w}(u) .
$$

By taking the average of the inequality (14) using the distribution $\pi_{C}$, we obtain the following inequality:

$$
\begin{aligned}
& \sum_{w \in C} \pi_{C}(w)\left(A_{H} D_{H}^{-1}-I\right)\left(\boldsymbol{p}_{w}\right)(\bar{C}) \\
& \leq \sum_{u \in C} \sum_{v \in \bar{C}} \frac{w_{\boldsymbol{p}_{w_{0}}}(u v)}{d_{u}}\left(\sum_{w \in C} \pi_{C}(w) \boldsymbol{p}_{w}\right)(u) .
\end{aligned}
$$

Now, we have

$$
\begin{aligned}
& \sum_{w \in C} \pi_{C}(w) \boldsymbol{p}_{w}(\bar{C})=\sum_{w \in C} \pi_{C}(w)\left(\frac{1-\alpha}{2 \alpha}\left(A_{H} D_{H}^{-1}-I\right) \boldsymbol{p}_{w}(\bar{C})\right) \\
& \leq \frac{1-\alpha}{2 \alpha} \sum_{u \in C} \sum_{v \in \bar{C}} \frac{w_{p_{w_{0}}}(u v)}{d_{u}}\left(\sum_{w \in C} \pi_{C}(w) p_{w}\right)(u) \quad \text { (by (9)) } \\
& \leq \frac{1-\alpha}{2 \alpha} \sum_{u \in C} \sum_{v \in \bar{C}} \frac{w_{p_{w_{0}}}(u v)}{d_{u}} \frac{d_{u}}{\operatorname{vol}(C)} \quad \text { (by the assumption) } \\
& \leq \frac{1-\alpha}{2 \alpha} \phi_{H}(C) \leq \frac{1}{2 \alpha} \phi_{H}(C) .
\end{aligned}
$$

\subsection{Proof of Theorem 9}

For a subset $C \subseteq V$ and $\alpha \in(0,1]$, we define a subset $C_{\alpha} \subseteq C$ as

$$
C_{\alpha}=\left\{v \in C \mid \operatorname{pr}_{\alpha}\left(\chi_{v}\right)(\bar{C}) \leq \frac{\phi_{H}(C)}{\alpha}\right\} .
$$

We here show the following from which Theorem 9 easily follows.

Theorem 11. Let $C^{*} \subseteq V$ be a set with $\phi_{H}=\phi_{H}\left(C^{*}\right)$ and assume $\operatorname{vol}\left(C^{*}\right) \leq \operatorname{vol}(V) / 2$. Suppose that $\alpha \leq 10 \phi_{H} \leq(1+\epsilon) \alpha$ and the condition (13) hold. Then, for any $v \in\left(C^{*}\right)_{\alpha}$, we have

$$
\phi_{H}^{1 / 2}\left(\operatorname{pr}_{\alpha}\left(\chi_{v}\right)\right)<\frac{20}{\sqrt{4-\epsilon}} \sqrt{3 \phi_{H} \log \left(\frac{40}{4-\epsilon}\right)} .
$$

Theorem 11 follows from Lemma 4 for $\mu=1 / 2$ and the following lemma. The proof of Lemma 12 is similar to the proof of Theorem 5.1 in [5] with Lemma 10. The detail is in Appendix C.

Lemma 12. Suppose a set $C \subseteq V$ and $\alpha>0$ satisfy the condition (13). Then, $\operatorname{vol}\left(C_{\alpha}\right) \geq \operatorname{vol}(C) / 2$ holds.

Proof of Theorem 11. By instantiating Lemma 12 with $C=C^{*}$ and $\alpha \leq 10 \phi_{H} \leq(1+\epsilon) \alpha$, we have

$$
\operatorname{pr}_{\alpha}\left(\chi_{v}\right)\left(C^{*}\right) \geq 1-\frac{1+\epsilon}{10}=\frac{9-\epsilon}{10}
$$


for $v \in\left(C^{*}\right)_{\alpha}$. Because $\operatorname{vol}\left(C^{*}\right) \leq \operatorname{vol}(V) / 2$,

$$
\operatorname{pr}_{\alpha}\left(\chi_{v}\right)\left(C^{*}\right)-\pi\left(C^{*}\right) \geq \frac{9-\epsilon}{10}-\frac{1}{2}=\frac{4-\epsilon}{10} .
$$

Instantiating Lemma 4 with $\mu=1 / 2, \delta=(4-\epsilon) / 10$, and $\alpha \leq 10 \phi_{H}$, we obtain

$$
\phi_{H}^{1 / 2}\left(\mathbf{p r}_{\alpha}\left(\chi_{v}\right)\right)<\frac{20}{\sqrt{4-\epsilon}} \sqrt{3 \phi_{H} \log \left(\frac{40}{4-\epsilon}\right)} .
$$

Proof of Theorem 9. The bound on conductance follows from Theorem 11 . The analysis on the time complexity is trivial.

\subsection{Sufficient conditions}

Here we discuss two useful sufficient conditions of the assumption (13) in Lemma 10. We give the proofs in Appendix D.

Lemma 13. Let $\alpha \in(0,1]$ and $C \subseteq V$ be a vertex set with $\operatorname{vol}(C) \leq$ $\operatorname{vol}(V) / 2$. If $\operatorname{pr}_{\alpha}\left(\chi_{v}\right)(v) \leq 1 / 2$ holds for every $v \in V$, then the condition (13) holds for any $v \in C \backslash C^{\circ}$.

LEMMA 14. Let $d_{\max }=\max _{v \in V}\left\{d_{v}\right\}$. If $\alpha \in(0,1]$ satisfies

$$
\alpha \leq\left(\frac{1}{2}-\frac{d_{\max }}{\operatorname{vol}(V)}\right)\left(1-\frac{d_{\max }}{\operatorname{vol}(V)}\right)^{-1},
$$

then the assumption (13) holds for any $v \in C \backslash C^{\circ}$.

Lemma 14 implies that, if $d_{\max } / \operatorname{vol}(V) \leq 1 / 4$ holds, then Lemma 10 holds for any $\alpha \leq 1 / 4$, which is usually the case in practice.

\section{EXPERIMENTAL EVALUATION}

In this section, we evaluate the performance of Algorithms 1 and 2 in terms of both the quality of solutions and running time.

Dataset. Table 1 lists real-world hypergraphs on which our experiments were conducted. Except for DBLP KDD, all hypergraphs were collected from $\mathrm{KONECT}^{1}$. As these are originally unweighted bipartite graphs, we transformed each of them into a hypergraph (with edge weights) as follows: We take the left-hand-side vertices in a given bipartite graph as the vertices in the corresponding hypergraph, and then for each right-hand-side vertex, we add a hyperedge (with weight one) consisting of the neighboring vertices in the bipartite graph. DBLP KDD is a coauthorship hypergraph, which we compiled from the DBLP dataset ${ }^{2}$. Specifically, the vertices correspond to the authors of the papers in KDD 1995-2019, while each hyperedge represents a paper, which contains the vertices corresponding to the authors of the paper.

As almost all hypergraphs generated as above were disconnected, we extracted the largest connected component in each hypergraph. All information in Table 1 is about the resulting hypergraphs.

Parameter setting. We explain the parameter setting of our algorithms. Throughout the experiments, we set $\epsilon=0.9$. In our implementation, to make the algorithms more scalable, we try to keep the PageRank vector sparse; that is, we round every PageRank value less than $10^{-5}$ down to zero in each iteration of the Euler method. As a preliminary experiment, using the two largest hypergraphs, we observed the effect of the parameters in the Euler

\footnotetext{
${ }^{1}$ http://konect.uni-koblenz.de/

2 https://dblp.uni-trier.de/
}

Table 1: Real-world hypergraphs used in our experiments.

\begin{tabular}{lrrrr}
\hline Name & $n$ & $m$ & $\sum_{v \in V} d_{v} / n$ & $\sum_{e \in E}|e| / m$ \\
\hline Graph products & 86 & 106 & 3.57 & 2.04 \\
Network theory & 330 & 299 & 3.06 & 3.04 \\
DBLP KDD & 5,590 & 2,719 & 1.99 & 4.00 \\
arXiv cond-mat & 13,861 & 13,571 & 3.87 & 3.05 \\
DBpedia Writers & 54,909 & 18,232 & 1.80 & 5.29 \\
YouTube Group Memberships & 88,490 & 21,974 & 3.24 & 12.91 \\
DBpedia Record Labels & 158,385 & 9,827 & 1.40 & 22.51 \\
DBpedia Genre & 253,968 & 4,934 & 1.80 & 92.84 \\
\hline
\end{tabular}

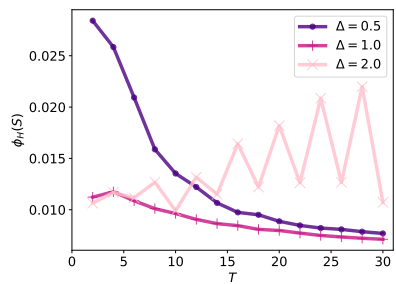

(a) DBpedia Record Labels

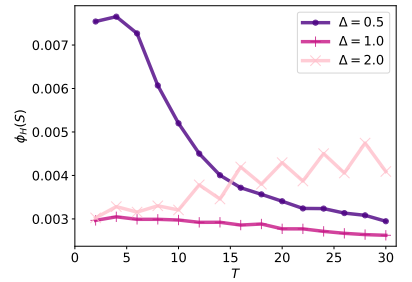

(b) DBpedia Genre
Figure 2: Effect of parameters $\Delta$ and $T$. Average values over 50 initial vertices are plotted.

method, $\Delta$ (i.e., time span) and $T$ (i.e., total time), in terms of the solution quality. Specifically, we selected 50 initial vertices uniformly at random, and for each of which, we ran Algorithm 1 with $\mu=1 / 2$ for each pair of parameters $(\Delta, T) \in\{0.5,1.0,2.0\} \times\{2,4, \ldots, 30\}$. The results are depicted in Figure 2. As can be seen, the effect of $\Delta$ is drastic. The performance with $\Delta=0.5$ is worse than that with $\Delta=1.0$, which implies that due to the above-mentioned sparsification of the PageRank vector, we have to spend some amount of time at once to obtain a non-negligible diffusion. Moreover, the performance with $\Delta=2.0$ is not stable, meaning that we should not use an unnecessarily large value for $\Delta$. The effect of $T$ is also significant; when $\Delta=0.5$ or 1.0 , the conductance of the output solution becomes smaller as $T$ increases. From these observations, in the following main experiments, we set $\Delta=1.0$ and $T=30$.

Baseline methods. In local clustering, we used two baselines called CLIQUE and STAR. CLIQUE first transforms a given hypergraph into a graph using the clique expansion, i.e., for each hyperedge $e \in E$ and each $u, v \in e$ with $u \neq v$, it adds an edge $u v$ of weight $w(e)$. Then CLIQUE computes personalized PageRank with some $\alpha$ using the power method and produces sweep cuts. Finally, it outputs the best subset among them, in terms of the conductance in the original hypergraph. STAR is an analogue of CLIQUE, which employs the star expansion alternatively, i.e., for each hyperedge $e \in E$, it introduces a new vertex $v_{e}$, and then for each vertex $u \in e$, it adds an edge $u v_{e}$ of weight $w(e) /|e|$. As the set of vertices has been changed in STAR, we leave out the additional vertices from the sweep cut. As for the power method, the initial (PageRank) vector is set to be $(1 / n, \ldots, 1 / n)$ and the stopping condition is set to be $\left\|x-x_{\text {prev }}\right\|_{1} \leq 10^{-8}$, where $x$ and $x_{\text {prev }}$ are vectors obtained in the current and previous iterations, respectively. Note that if we employ $A_{\text {cand }}$ as the candidate set of $\alpha$, the power method requires numerous number of iterations. This is because 


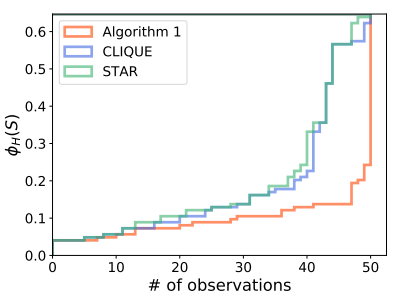

(a) Graph products

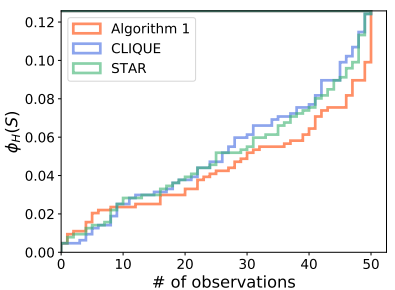

(e) DBpedia Writers

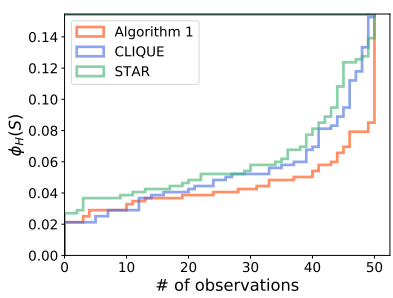

(b) Network theory

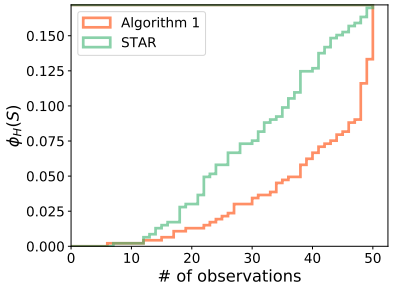

(f) YouTube Group Memberships

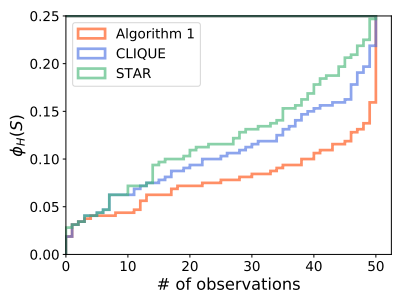

(c) DBLP KDD

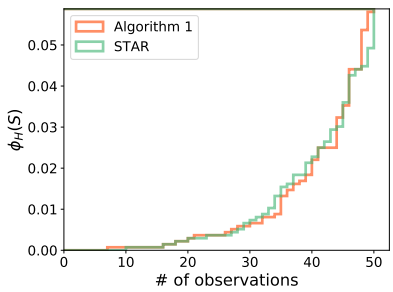

(g) DBpedia Record Labels

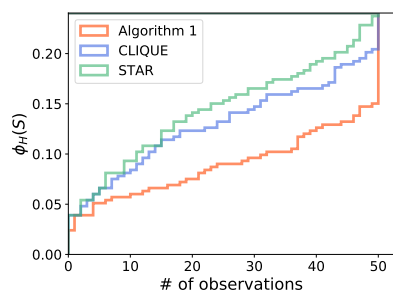

(d) arXiv cond-mat

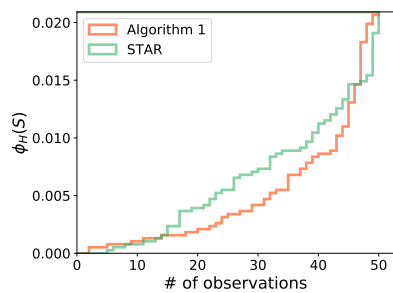

(h) DBpedia Genre

Figure 3: Comparison of the solution quality of Algorithm 1 with those of CLIQUE and STAR.

$A_{\text {cand }}$ contains a lot of small values, e.g., $\frac{w_{\min }}{w_{\max } \sum_{e \in E}|e|}$. Hence, we just used $\alpha=0.05,0.10, \ldots, 0.95$ in our experiments.

In global clustering, we used the well-known hypergraph clustering package called $\mathrm{hMETIS}{ }^{3}$ as well as the global counterparts of CLIQUE and STAR, which can be obtained in the same way as that of our algorithms. Note that hMETIS has a lot of parameters; among those, the parameters UBfactor, Nruns, CType, RType, and Vcycle may affect the solution quality and running time. To obtain a high quality solution, we run the algorithm for each of (UBfactor, Nruns, CType, RType, Vcycle) $\in\{5,10, \ldots, 45\} \times\{10\} \times$ $\{1,2,3,4,5\} \times\{1,2,3\} \times\{0,1,2,3\}$ to obtain vertex sets, and then return the one with the smallest conductance. We used hMETIS 1.5.3, the latest stable release. Finally, we remark that hMETIS is not applicable to local clustering because we cannot specify the volume of a subset containing the specified vertex.

\subsection{Local clustering}

First, we focus on local clustering and evaluate the performance of Algorithm 1. To this end, for each hypergraph in Table 1, we selected 50 initial vertices uniformly at random, and for each of which, we ran Algorithm 1, CLIQUE, and STAR. To focus on local structure, Algorithm 1 employs $\mu=1 / 10$, and so do CLIQUE and STAR in themselves. The code is written in C\#, which was conducted on a machine with $2.8 \mathrm{GHz}$ CPU and 16 GB RAM.

The quality of solutions are illustrated in Figure 3 . The results for CLIQUE are missing for the three largest hypergraphs because it could not be performed due to the memory limit. In those plots, once we fix a value in the vertical axis, we can see the cumulative number of solutions with the conductance less than or equal to the value fixed; thus, an algorithm drawing a line with a lower right position has a better performance. As can be seen, Algorithm 1 outperforms CLIQUE and STAR. In fact, Algorithm 1 almost always

\footnotetext{
${ }^{3}$ http://glaros.dtc.umn.edu/gkhome/metis/hmetis/overview
}

Table 2: Running time(s) of local algorithms.

\begin{tabular}{lrrr}
\hline Name & Algorithm 1 & CLIQUE & STAR \\
\hline Graph products & 0.02 & $\mathbf{0 . 0 1}$ & 0.03 \\
Network theory & $\mathbf{0 . 0 6}$ & 0.18 & 0.11 \\
DBLP KDD & $\mathbf{1 . 4 2}$ & 1.84 & 1.54 \\
arXiv cond-mat & 5.43 & $\mathbf{4 . 8 4}$ & 5.70 \\
DBpedia Writers & $\mathbf{7 . 0 7}$ & 77.98 & 17.50 \\
YouTube Group Memberships & 93.67 & OM & $\mathbf{5 7 . 6 4}$ \\
DBpedia Record Labels & $\mathbf{3 6 . 6 9}$ & OM & 44.18 \\
DBpedia Genre & $\mathbf{8 5 . 8 6}$ & OM & 97.47 \\
\hline
\end{tabular}

has a larger cumulative number with a fixed conductance value. It can be observed that CLIQUE performs better than STAR.

Table 2 presents the running time of the algorithms, where we have the average values over 50 initial vertices. The best results among the algorithms are written in bold. As is evident, Algorithm 1 is much more scalable than CLIQUE and comparable to STAR.

\subsection{Global clustering}

Next we focus on global clustering and evaluate the performance of Algorithm 2. As can be inferred from Table 2, Algorithm 2 itself does not scale for large hypergraphs. Therefore, we modified the algorithm as follows: we selected 50 initial vertices uniformly at random, and replace $V$ with the set of selected vertices in Line 2 (in Algorithm 2). We modified the global counterparts of CLIQUE and STAR in the same way. To compare the performance of the algorithms fairly, we used the same initial vertices for those three algorithms. The code is again written in C\#, which was conducted on the above-mentioned machine.

The results are summarized in Table 3, where the best results are again written in bold. As for the solution quality, Algorithm 2 is one of the best choices, but unlike the local setting, STAR performs well. 
Table 3: Performance of Algorithm 2, CLIQUE, STAR, and hMETIS.

\begin{tabular}{|c|c|c|c|c|c|c|c|c|}
\hline \multirow[b]{2}{*}{ Name } & \multicolumn{2}{|c|}{ Algorithm 2} & \multicolumn{2}{|c|}{ CLIQUE } & \multicolumn{2}{|c|}{ STAR } & \multicolumn{2}{|c|}{ hMETIS } \\
\hline & $\phi_{H}(S)$ & Time(s) & $\phi_{H}(S)$ & Time(s) & $\phi_{H}(S)$ & Time(s) & $\phi_{H}(S)$ & Time(s) \\
\hline Graph products & 0.0234 & 1.01 & 0.0246 & 0.70 & 0.0288 & 1.47 & 0.0234 & 15.30 \\
\hline Network theory & 0.0064 & 3.03 & 0.0062 & 8.57 & 0.0074 & 5.15 & 0.0061 & 24.82 \\
\hline DBLP KDD & 0.0116 & 68.91 & 0.0116 & 89.24 & 0.0116 & 74.98 & 0.0236 & 107.03 \\
\hline arXiv cond-mat & 0.0127 & 251.81 & 0.0127 & 237.31 & 0.0127 & 281.37 & 0.0259 & 418.08 \\
\hline DBpedia Writers & 0.0069 & 353.60 & 0.0043 & $3,812.72$ & 0.0048 & 856.85 & 0.0010 & 721.36 \\
\hline YouTube Group Memberships & 0.0003 & $4,735.28$ & - & $\mathrm{OM}$ & 0.0003 & $2,779.52$ & 0.0029 & $3,696.24$ \\
\hline DBpedia Record Labels & 0.0002 & $1,766.34$ & - & $\mathrm{OM}$ & 0.0002 & $2,151.80$ & 0.0052 & $1,655.88$ \\
\hline DBpedia Genre & 0.0001 & $4,320.72$ & - & $\mathrm{OM}$ & 0.0001 & $4,779.95$ & 0.0023 & $8,670.49$ \\
\hline
\end{tabular}

In fact, STAR achieves the same performance as that of Algorithm 2 for the three largest hypergraphs. On the other hand, the performance of hMETIS is unfavorable. In our experiments, hMETIS was performed with almost all possible parameter settings; therefore, it is unlikely that we can obtain a comparable solution using hMETIS. The trend of the results for the running time is similar to that of the local setting; Algorithm 1 is much more scalable than CLIQUE and comparable to STAR.

\section{CONCLUSION}

In this work, we have developed local and global clustering algorithms based on PageRank for hypergraphs, recently introduced by $\mathrm{Li}$ and Milenkovic [22]. To the best of our knowledge, ours are the first practical algorithms that have theoretical guarantees on the conductance of the output vertex set. By experiment, we confirmed the usefulness of our clustering algorithms in practice.

\section{ACKNOWLEDGMENTS}

The authors thank Yu Kitabeppu for helpful comments. This work was done while A.M. was at RIKEN AIP, Japan. This work was supported by JSPS KAKENHI Grant Numbers 18H05291 and 19K20218.

\section{REFERENCES}

[1] Sameer Agarwal, Kristin Branson, and Serge Belongie. 2006. Higher order learning with graphs. In ICML. 17-24.

[2] Sameer Agarwal, Jongwoo Lim, Lihi Zelnik-Manor, Pietro Perona, David Kriegman, and Serge Belongie. 2005. Beyond pairwise clustering. In CVPR. 838-845.

[3] Noga Alon. 1986. Eigenvalues and expanders. Combinatorica 6, 2 (1986), 83-96.

[4] Noga Alon and V. D. Milman. 1985. $\lambda_{1}$, Isoperimetric inequalities for graphs, and superconcentrators. fournal of Combinatorial Theory, Series B 38, 1 (1985), 73-88.

[5] Reid Andersen, Fan Chung, and Kevin Lang. 2007. Using PageRank to locally partition a graph. Internet Mathematics 4, 1 (2007), 35-64.

[6] Reid Andersen and Yuval Peres. 2009. Finding sparse cuts locally using evolving sets. In STOC. 235-244.

[7] Marianna Bolla. 1993. Spectra, Euclidean representations and clusterings of hypergraphs. Discrete Mathematics 117 (1993), 19-39.

[8] T-H Hubert Chan, Anand Louis, Zhihao Gavin Tang, and Chenzi Zhang. 2018 Spectral properties of hypergraph Laplacian and approximation algorithms. 7 . ACM 65, 3 (2018), 15-48.

[9] I Chien, Chung-Yi Lin, and I-Hsiang Wang. 2018. Community detection in hypergraphs: Optimal statistical limit and efficient algorithms. In AISTATS. 871879.

[10] Fan Chung. 2007. The heat kernel as the pagerank of a graph. Proceedings of the National Academy of Sciences of the United States of America 104, 50 (2007), 19735-19740.

[11] Pedro F. Felzenszwalb and Daniel P. Huttenlocher. 2004. Efficient graph-based image segmentation. International fournal of Computer Vision 59, 2 (2004), 167 181
[12] Santo Fortunato. 2010. Community detection in graphs. Physics Reports 486, 3-5 (2010), 75-174.

[13] Kaito Fujii, Tasuku Soma, and Yuichi Yoshida. 2018. Polynomial-time algorithms for submodular Laplacian systems. arXiv preprint, arXiv:1803.10923 (2018).

[14] Shayan Oveis Gharan and Luca Trevisan. 2012. Approximating the expansion profile and almost optimal local graph clustering. In FOCS. 187-196.

[15] Debarghya Ghoshdastidar and Ambedkar Dukkipati. 2014. Consistency of spectral partitioning of uniform hypergraphs under planted partition model. In NIPS. 397-405.

[16] Masahiro Ikeda, Atsushi Miyauchi, Yuuki Takai, and Yuichi Yoshida. 2018. Finding Cheeger cuts in hypergraphs via heat equation. arXiv preprint arXiv:1809.04396 (2018).

[17] Glen Jeh and Jennifer Widom. 2003. Scaling personalized web search. In WWW. 271-279.

[18] Kyle Kloster and David F. Gleich. 2014. Heat kernel based community detection. In KDD. 1386-1395.

[19] Yukio Komura. 1967. Nonlinear semi-groups in Hilbert space. Fournal of the Mathematical Society of Japan 19, 4 (1967), 493-507.

20] Tsz C. Kwok, Lap C. Lau, and Yin T. Lee. 2017. Improved Cheeger's inequality and analysis of local graph partitioning using vertex expansion and expansion profile. SIAM F. Comput. 46, 3 (2017), 890-910.

[21] Marius Leordeanu and Cristian Sminchisescu. 2012. Efficient hypergraph clustering. In AISTATS. 676-684

[22] Pan Li and Olgica Milenkovic. 2018. Submodular hypergraphs: p-Laplacians, Cheeger inequalities and spectral clustering. In ICML. 3014-3023.

[23] Hairong Liu, Longin J. Latecki, and Shuicheng Yan. 2010. Robust clustering as ensembles of affinity relations. In NIPS. 1414-1422.

[24] László Lovász and Miklós Simonovits. 1990. The mixing rate of Markov chains, an isoperimetric inequality, and computing the volume. In FOCS. 346-354.

[25] László Lovász and Miklós Simonovits. 1993. Random walks in a convex body and an improved volume algorithm. Random Structures \& Algorithms 4, 4 (1993), 359-412.

[26] Isao Miyadera. 1992. Nonlinear Semigroups. Translations of Mathematical Monographs, Vol. 109. American Mathematical Society.

[27] Juan Peypouquet and Sylvain Sorin. 2010. Evolution Equations for Maximal Monotone Operators: Asymptotic Analysis in Continuous and Discrete Time. Journal of Convex Analysis 17, 3\&4 (2010), 1113-1163.

[28] J. A. Rodri'guez. 2002. On the Laplacian eigenvalues and metric parameters of hypergraphs. Linear and Multilinear Algebra 50, 1 (2002), 1-14.

[29] Samuel Rota Bulo and Marcello Pelillo. 2013. A game-theoretic approach to hypergraph clustering. IEEE Transactions on Pattern Analysis and Machine Intelligence 35, 6 (2013), 1312-1327.

[30] Daniel A. Spielman and Shang-Hua Teng. 2013. A local clustering algorithm for massive graphs and its application to nearly linear time graph partitioning. SIAM 7. Comput. 42, 1 (2013), 1-26.

[31] Yuichi Yoshida. 2016. Nonlinear Laplacian for digraphs and its applications to network analysis. In WSDM. 483-492.

[32] Yuichi Yoshida. 2019. Cheeger inequalities for submodular transformations. In SODA. 2582-2601.

[33] Dengyong Zhou, Jiayuan Huang, and Bernhard Schölkopf. 2005. Beyond pairwise classification and clustering using hypergraphs. Max Plank Institute for Biological Cybernetics, Tübingen, Germany (2005).

[34] Dengyong Zhou, Jiayuan Huang, and Bernhard Schölkopf. 2007. Learning with hypergraphs: Clustering, classification, and embedding. In NIPS. 1601-1608.

[35] Jason Y. Zien, Martin D. F. Schlag, and Pak K. Chan. 1999. Multilevel spectral hypergraph partitioning with arbitrary vertex sizes. IEEE Transactions on Pattern Analysis and Machine Intelligence 18, 9 (1999), 1389-1399. 


\section{A PROOFS OF SECTION 4.1}

Proof of Theorem 1. We first prove the former claim. In [16], it was proven that $\mathcal{L}_{H}$ is a maximal monotone operator. (We omit the definition here because we do not need it). Then, by the general theory of maximal monotone operators $[19,26]$, the operator $I+$ $\lambda \mathcal{L}_{H}$ is injective for any $\lambda>0$. Hence, its inverse $J_{\lambda}:=\left(I+\lambda \mathcal{L}_{H}\right)^{-1}$ is single-valued, and is called a resolvent of $\mathcal{L}_{H}$. Because the image of $\mathcal{L}_{H}$ is $\mathbb{R}^{V}$, for any $s \in \mathbb{R}^{V}$, there exists a unique vector $J_{\lambda}(\boldsymbol{s})$, which is a solution to (3) when $\lambda=(1-\alpha) / 2 \alpha>0$.

We now prove the latter claim. For $p=\operatorname{pr}_{\alpha}(s)$, we have

$$
p+\frac{1-\alpha}{2 \alpha} \mathcal{L}_{H_{p}} p=s,
$$

where $H_{p}$ is the graph obtained from $H$ and $\boldsymbol{p}$, as defined in Section 3.3. This implies that $\boldsymbol{p}$ is a classical PPR on $H_{\boldsymbol{p}}$, and hence the claim holds by the Perron-Frobenius theorem for the matrix $\alpha \boldsymbol{s} \mathbf{1}^{\top}+(1-\alpha) W_{H_{p}}$.

Proof of Propostion 2. As mentioned in the proof of Theorem 1, the PPR $\operatorname{pr}_{\alpha}(s)$ is equal to a resolvent $J_{\lambda}(s)$ for $\lambda=(1-\alpha) / 2 \alpha$. Thus, it suffices to prove the continuity of the resolvent $J_{\lambda}(s)$ with respect to $\lambda>0$. By [26], for $\lambda \geq \mu>0$, the resolvents satisfy the following inequality:

$$
J_{\lambda}(s)=J_{\mu}\left(\frac{\mu}{\lambda} s+\frac{\lambda-\mu}{\lambda} J_{\lambda}(s)\right) .
$$

On the other hand, it is well known that $J_{\lambda}$ is a non-expansive map, i.e., for any $s, t \in \mathbb{R}^{V}$ and any $\lambda>0,\left\|J_{\lambda}(\boldsymbol{s})-J_{\lambda}(\boldsymbol{t})\right\|_{D_{H}^{-1}} \leq\|\boldsymbol{s}-\boldsymbol{t}\|_{D_{H}^{-1}}$ where $\|\boldsymbol{x}\|_{D_{H}^{-1}}=\sqrt{\boldsymbol{x}^{\top} D_{H}^{-1} \boldsymbol{x}}$. By these relations, we can show the following:

$$
\begin{aligned}
& \left\|J_{\lambda}(\boldsymbol{s})-J_{\mu}(\boldsymbol{s})\right\|_{D_{H}^{-1}} \leq\left\|J_{\mu}\left(\frac{\mu}{\lambda} \boldsymbol{s}+\frac{\lambda-\mu}{\lambda} J_{\lambda}(\boldsymbol{s})\right)-J_{\mu}(\boldsymbol{s})\right\|_{D_{H}^{-1}} \\
& \leq\left\|\frac{\mu}{\lambda} \boldsymbol{s}+\frac{\lambda-\mu}{\lambda} J_{\lambda}(\boldsymbol{s})-\boldsymbol{s}\right\|_{D_{H}^{-1}} \leq \frac{\lambda-\mu}{\lambda}\left\|J_{\lambda}(\boldsymbol{s})-\boldsymbol{s}\right\|_{D_{H}^{-1}} .
\end{aligned}
$$

Hence, $\lim _{\lambda \rightarrow \mu} J_{\lambda}(s)=J_{\mu}(s)$.

To prove Proposition 3, we prepare the following lemma:

LEMmA 15. For $\beta:=2 \alpha /(1+\alpha)$, we have

$$
\beta\left(s-\operatorname{pr}_{\alpha}(s)\right)-(1-\beta) \mathcal{L}_{H}\left(\mathbf{p r}_{\alpha}(s)\right)=0 .
$$

Proof of Lemma 15 . Let $\boldsymbol{p}=\operatorname{pr}_{\alpha}(\boldsymbol{s})$. Note that $(1-\alpha) / 2 \alpha=$ $1 / \beta-1$. Then by (3) and easy calculation, we have

$$
\left(I+\frac{1-\alpha}{2 \alpha} \mathcal{L}_{H}\right)(\boldsymbol{p}) \ni \boldsymbol{s} \Leftrightarrow \beta(\boldsymbol{s}-\boldsymbol{p})-(1-\beta) \mathcal{L}_{H}(\boldsymbol{p}) \ni \mathbf{0} .
$$

As $\boldsymbol{p}$ is unique by Theorem 1, we can replace the inclusion with equality.

Proof of Proposition 3. Let $\boldsymbol{p}=\operatorname{pr}_{\alpha}\left(\chi_{u}\right)$. Because $\boldsymbol{p}$ is a distribution by the latter claim of Theorem 1, we have $p(v) \geq 0$ for any $v \in V$. Then by Lemma 15 , we have

$$
\boldsymbol{p}=\chi_{u}-\frac{1-\beta}{\beta} \mathcal{L}_{H_{p}} \boldsymbol{p} \geq \mathbf{0}
$$

Hence for $v \neq u$, we have $-\mathcal{L}_{H_{p}} p(v) \geq 0$. Note that $-\mathcal{L}_{H_{p}} p(v)$ is equal to the difference between the heat diffusing into $v$ and that diffusing out from $v$ in the heat equation:

$$
\frac{d \rho_{t}}{d t}=-\mathcal{L}_{H_{p}} \rho_{t}
$$

Because the heat diffuses to the stationary distribution $\pi_{V}$, the non-negativity of $-\mathcal{L}_{H_{p}} \boldsymbol{p}(v)$ implies $\boldsymbol{p}(v) \leq \boldsymbol{\pi}_{V}(v)$.

When $v=u$, we have $\boldsymbol{p}(u)-\chi_{u}(u) \leq 0$. By a similar argument, we have the desired inequality.

\section{B PROOF OF LEMMA 4}

\section{B.1 Setup}

Before proving Lemma 4, we first introduce several notations. Let $G=(V, E, w)$ be an undirected graph. For a subset $S \subseteq V$, we define two sets of ordered pairs of vertices as follows:

$$
\operatorname{in}(S):=\{(u, v) \in V \times S\}, \operatorname{out}(S):=\{(u, v) \in S \times V\} .
$$

For an ordered pair $(u, v) \in V \times V$ and a distribution $\boldsymbol{p} \in \mathbb{R}^{V}$, we set $\boldsymbol{p}(u, v):=\frac{w(u v)}{d_{u}} \boldsymbol{p}(u)$. For a set of ordered pairs of vertices $A \subseteq V \times V$ and any distribution $\boldsymbol{p} \in \mathbb{R}^{V}$, we set $\boldsymbol{p}(A):=\sum_{(u, v) \in A} \boldsymbol{p}(u, v)$. Then, by a similar argument for $\boldsymbol{p}$ and $H_{\boldsymbol{p}}$ to [5], we have the following:

Lemma 16. Let $H=(V, E, w)$ be a hypergraph, $\boldsymbol{p} \in \mathbb{R}^{V}$ be a distribution, $H_{p}=\left(V, E_{p}, w_{p}\right)$ be a graph as defined in Section 3, and $S \subseteq V$. Then, we have

$$
W_{H_{p}}(\boldsymbol{p})(S)=\frac{1}{2}(\boldsymbol{p}(\operatorname{in}(S) \cup \operatorname{out}(S))+\boldsymbol{p}(\operatorname{in}(S) \cap \operatorname{out}(S))),
$$

where $\boldsymbol{p}(\cdot)$ is defined using the graph $\mathrm{H}_{\boldsymbol{p}}$.

\section{B.2 Lovász-Simonovic function for weighted graphs}

Next, we introduce a weighted version of the Lovász-Simonovic function $\boldsymbol{p}[x][24,25]$. For a distribution $\boldsymbol{p} \in \mathbb{R}^{V}$, we define $\boldsymbol{p}[x]$ on $x \in[0, \operatorname{vol}(V)]$ as follows: If $x=\operatorname{vol}\left(S_{j}^{p}\right)$ for some $j=0,1, \ldots, n$, we define $\boldsymbol{p}[x]:=\boldsymbol{p}\left(S_{j}^{p}\right)$. For general $x \in[0, \operatorname{vol}(V)]$, we extend $p[x]$ as a piecewise linear function. More specifically, if $x$ satisfies $\operatorname{vol}\left(S_{j}^{\boldsymbol{p}}\right) \leq x<\operatorname{vol}\left(S_{j+1}^{\boldsymbol{p}}\right)$, then $\boldsymbol{p}[x]$ is defined as

$$
\boldsymbol{p}[x]=\boldsymbol{p}\left(S_{j}^{\boldsymbol{p}}\right)+\frac{\boldsymbol{p}\left(v_{j+1}\right)}{d_{v_{j+1}}}\left(x-\operatorname{vol}\left(S_{j}^{\boldsymbol{p}}\right)\right) .
$$

We remark that the function $p[x]$ is concave. Then, an argument similar to that of [5] with Lemma 16 implies the following lemma:

LEMMA 17. For $\boldsymbol{p}=\operatorname{pr}_{\alpha}(\boldsymbol{s})$ and $j=1,2, \ldots, n-1$, we have

$$
\begin{aligned}
& \boldsymbol{p}\left[\operatorname{vol}\left(S_{j}^{\boldsymbol{p}}\right)\right] \leq \alpha \boldsymbol{s}\left[\operatorname{vol}\left(S_{j}^{\boldsymbol{p}}\right)\right]+ \\
& \quad+(1-\alpha) \frac{1}{2}\left(\boldsymbol{p}\left[\operatorname{vol}\left(S_{j}^{\boldsymbol{p}}\right)-\left|\partial\left(S_{j}^{\boldsymbol{p}}\right)\right|\right]+\boldsymbol{p}\left[\operatorname{vol}\left(S_{j}^{\boldsymbol{p}}\right)+\left|\partial\left(S_{j}^{\boldsymbol{p}}\right)\right|\right]\right) .
\end{aligned}
$$

\section{B.3 A mixing result for PPR and the proof of Lemma 4}

Next, we show a local version of a mixing result for PPR. A global version for graphs was proven in [5]. 
Theorem 18. Let $s$ be a distribution and $\phi \in[0,1], \mu \in(0,1 / 2]$ be any constants. We set $\boldsymbol{p}=\mathrm{pr}_{\alpha}(\mathbf{s})$. Let $\ell_{\mu} \in\{1,2, \ldots, n\}$ be the unique integer such that $\operatorname{vol}\left(S_{\ell_{\mu}-1}^{p}\right)<\mu \cdot \operatorname{vol}(V) \leq \operatorname{vol}\left(S_{\ell_{\mu}}^{p}\right)$. We assume that $\phi_{H}\left(S_{j}^{p}\right) \geq \phi$ holds for any $j=1,2, \ldots, \ell_{\mu}$. Then, for any $S \subseteq V$ such that $\operatorname{vol}(S) / \operatorname{vol}(V) \leq \mu$ and any $t \in \mathbb{Z}_{+}$, the following holds:

$$
\boldsymbol{p}(S)-\boldsymbol{\pi}_{V}(S) \leq \alpha t+\sqrt{\operatorname{vol}(S)}\left(1-\frac{\phi^{2}}{8}\right)^{t} .
$$

Proof. We can show this theorem by an argument similar to that of [5]. Here, we show a sketch of the proof.

We define a function $f_{t}(x)$ on $x \in\left[0, \operatorname{vol}\left(S_{\ell_{\mu}}^{p}\right)\right]$ by

$$
f_{t}(x):=\alpha t+\sqrt{\min \{x, \operatorname{vol}(V)-x\}}\left(1-\frac{\phi^{2}}{8}\right)^{t} .
$$

Because $\boldsymbol{p}(S) \leq \boldsymbol{p}[\operatorname{vol}(S)]$ for any vertex set $S \subseteq V$, if for a vertex set $S \subseteq V$ such that $\operatorname{vol}(S) / \operatorname{vol}(V) \leq \mu$,

$$
p[x]-\frac{x}{\operatorname{vol}(V)} \leq f_{t}(x)
$$

holds for $x=\operatorname{vol}(S)$, then the inequality (16) for $S$ follows.

To prove Theorem 18, by induction on $t$, we show that if a sweep cut $S_{j}^{\boldsymbol{p}}$ satisfies $\phi_{H}\left(S_{j}^{\boldsymbol{p}}\right) \geq \phi$, then the inequality (17) holds for $x_{j}=\operatorname{vol}\left(S_{j}^{p}\right)$ and any $t \in \mathbb{Z}_{+}$as follows:

(1) We prove that the inequality (17) holds for $t=0$ and any $S \subseteq V$.

(2) We assume that the inequality (17) holds for $t=t_{0}$ and $x_{j}=$ $\operatorname{vol}\left(S_{j}^{p}\right)$. Then, by Lemma 17 , the concavity of $f_{t}\left(x_{j}\right)$, and the piecewise linearity of $\boldsymbol{p}\left[x_{j}\right]-x_{j} / \operatorname{vol}(V)$, we prove that if $S_{j}^{p}$ satisfies $\phi_{H}\left(S_{j}^{\boldsymbol{p}}\right) \geq \phi$, then the inequality (17) holds for $x_{j}=$ $\operatorname{vol}\left(S_{j}^{p}\right)$ and $t=t_{0}+1$.

This argument implies that if any sweep cut $S_{j}^{p}\left(j=1,2, \ldots, \ell_{\mu}\right)$ satisfies $\phi_{H}\left(S_{j}^{\boldsymbol{p}}\right) \geq \phi$, the inequality (17) holds for $x_{j}=\operatorname{vol}\left(S_{j}^{\boldsymbol{p}}\right)$ for all $j \in\left\{0,1, \ldots, \ell_{\mu}\right\}$ and any $t \in \mathbb{Z}_{+}$. Then, by the concavity of $f_{t}(x)$ and the piecewise linearity of $p[x]-x / \operatorname{vol}(V)$ again, the inequality (17) holds for any $x \in[0, \mu \cdot \operatorname{vol}(V)]$ and any $t \in \mathbb{Z}_{+}$. Because $\boldsymbol{p}(S) \leq \boldsymbol{p}[\operatorname{vol}(S)]$, we obtain the inequality (16) for any $S \subseteq V$ with $\operatorname{vol}(S) / \operatorname{vol}(V) \leq \mu$.

We next prove the following as a consequence of Theorem 18.

LeMmA 19. If there is a vertex set $S \subseteq V$ and a constant $\delta \geq$ $4 / \sqrt{\operatorname{vol}(V)}$ such that $\operatorname{vol}(S) / \operatorname{vol}(V) \leq \mu$ and $\operatorname{pr}_{\alpha}(s)(S)-\pi_{V}(S)>\delta$, then the following inequality holds:

$$
\phi_{H}^{\mu}\left(\mathbf{p r}_{\alpha}(\mathbf{s})\right)<\sqrt{\frac{12 \alpha \log (\operatorname{vol}(V))}{\delta}} .
$$

Proof. We apply Theorem 18 for $\phi=\phi_{H}^{\mu}\left(\operatorname{pr}_{\alpha}(s)\right)$. Then, because every sweep cut $S_{j}^{p}(j=1,2, \ldots, \ell)$ satisfies the assumption $\phi_{H}\left(S_{j}^{p}\right) \geq \phi$ in Theorem 18, any vertex set $S \subseteq V$ with $\operatorname{vol}(S) / \operatorname{vol}(V) \leq \mu$ satisfies the inequality (16) for any $t \in \mathbb{Z}_{+}$. As in the argument of [5], we take $t$ as

$$
t=\left\lceil\frac{8}{\phi^{2}} \log \left(\frac{4 \sqrt{\operatorname{vol}(S)}}{\delta}\right)\right\rceil \leq \frac{9}{\phi^{2}} \log \left(\frac{4 \sqrt{\operatorname{vol}(S)}}{\delta}\right) .
$$

Here, $\lceil x\rceil=\min \{a \in \mathbb{Z} \mid x \leq a\}$. Then by the inequality (16), we have

$$
\boldsymbol{p}(S)-\boldsymbol{\pi}_{V}(S) \leq \alpha \frac{9}{\phi^{2}} \log \left(\frac{4 \sqrt{\operatorname{vol}(S)}}{\delta}\right)+\frac{\delta}{4} .
$$

By the assumption $\delta<\boldsymbol{p}(S)-\boldsymbol{\pi}_{V}(S)$ and rearranging the obtained inequality, we have the desired inequality (18).

Finally, we deduce Lemma 4 from Lemma 19.

Proof of Lemma 4. The statement of Lemma 19 is independent of the normalization of the weight function $w: E \rightarrow \mathbb{R}_{\geq 0}$, except for the factor $\operatorname{vol}(V)$. Hence, for the hypergraph $H^{c}=(V, E, c w)$ scaled by $c>0$, the similar statement to Lemma 19 obtained by replacing $\operatorname{vol}(V)$ with $c \cdot \operatorname{vol}(V)$ also holds. This means that the right hand side of (18) can be decreased as long as the assumption $\delta \geq$ $4 / \sqrt{c \cdot \operatorname{vol}(V)}$ holds. Then, we remark that the obtained conductance $\phi_{H^{c}}^{\mu}\left(\mathbf{p r}_{\alpha}(s)\right)$ is independent of $c$. As a consequence of this argument, if a hypergraph $H$ satisfies the assumption of Lemma 19, then, Lemma 19 holds for $H^{c}$ for any $c$ that satisfies the assumption $\delta \geq 4 / \sqrt{c \cdot \operatorname{vol}(V)}$, hence also for $c \cdot \operatorname{vol}(V)=4^{2} / \delta^{2}$. However, the left hand side of (18) for $H$ is the same as that for $H^{c}$. This implies the inequality (7).

\section{PROOF OF LEMMA 12}

Proof of Lemma 12. Let $\boldsymbol{p}_{v}=\operatorname{pr}_{\alpha}\left(\chi_{v}\right)$ for $v \in V$. We consider a random variable $X:=p_{v}(\bar{C})$, where $v \in V$ is sampled according to the distribution $\pi_{C}$. Then by Lemma 10 , we have

$$
\mathrm{E}_{\boldsymbol{\pi}_{C}}[X]=\sum_{v \in V} \boldsymbol{\pi}_{C}(v) \boldsymbol{p}_{v}(\bar{C}) \leq \frac{\phi_{H}(C)}{2 \alpha} .
$$

By Markov's inequality, we have

$$
\operatorname{Pr}\left[v \notin C_{\alpha}\right] \leq \operatorname{Pr}\left[X>2 \mathrm{E}_{\pi_{C}}[X]\right] \leq \frac{1}{2} .
$$

This implies that $\operatorname{vol}\left(C_{\alpha}\right)$ is larger than $\operatorname{vol}(C) / 2$.

\section{PROOFS OF SUFFICIENT CONDITIONS}

Proof of Lemma 13. Let $\boldsymbol{p}_{v}=\operatorname{pr}_{\alpha}\left(\chi_{v}\right)$ for $v \in V$. For a vertex $v \in V$, we have

$$
\boldsymbol{p}_{v}(v) \leq \frac{1}{2} \leq 1-\frac{\operatorname{vol}(C)}{\operatorname{vol}(V)}
$$

Hence by multiplying both sides by $\pi_{C}(v)=d_{v} / \operatorname{vol}(C)$ and transposing a term, we have

$$
\boldsymbol{\pi}_{C}(v) \boldsymbol{p}_{v}(v)+\boldsymbol{\pi}_{V}(v) \leq \boldsymbol{\pi}_{C}(v) .
$$

Now, we have

$$
\begin{aligned}
& \sum_{u \in C} \boldsymbol{\pi}_{C}(u) \boldsymbol{p}_{u}(v)=\pi_{C}(v) \boldsymbol{p}_{v}(v)+\sum_{u \in C \backslash\{v\}} \pi_{C}(u) \boldsymbol{p}_{u}(v) \\
& \leq \boldsymbol{\pi}_{C}(v) \boldsymbol{p}_{v}(v)+\sum_{u \in C \backslash\{v\}} \boldsymbol{\pi}_{C}(u) \boldsymbol{\pi}_{V}(v) \quad \text { (by Proposition 3) } \\
& \leq \boldsymbol{\pi}_{C}(v) \boldsymbol{p}_{v}(v)+\pi_{V}(v) \leq \boldsymbol{\pi}_{C}(v) .
\end{aligned}
$$

This concludes the proof. 
Proof of Lemma 14. Let $\boldsymbol{p}=\operatorname{pr}_{\alpha}\left(\chi_{v}\right)$. By an argument similar to the proof of Lemma 10 for the case $C=\{v\}$, we have

$$
\alpha(1-\boldsymbol{p}(v))=(1-\alpha) \boldsymbol{p}(v)-(1-\alpha) \sum_{u \in \bar{C}, u \neq v} \frac{w_{p}(v u)}{d_{u}} \boldsymbol{p}(u) .
$$

Then, we have

$$
p(v)=\alpha+(1-\alpha) \sum_{u \in \bar{C}, u \neq v} \frac{w_{p}(v u)}{d_{u}} \boldsymbol{p}(u) .
$$

Because $\boldsymbol{p}(u) \leq d_{u} / \operatorname{vol}(V)$ holds for any $u \neq v$ by Proposition 3, we have

$$
p(v) \leq \alpha+(1-\alpha) \sum_{u \in \bar{C}, u \neq v} \frac{w_{p}(v u)}{d_{u}} \frac{d_{u}}{\operatorname{vol}(V)} \leq \alpha+(1-\alpha) \frac{d_{\max }}{\operatorname{vol}(V)}
$$

By combining this inequality with Lemma 13 , if $\alpha+(1-\alpha) d_{\max } / \operatorname{vol}(V) \leq$ $1 / 2$, then $\alpha$ satisfies the condition (13) for any $v \in C \backslash C^{\circ}$. 